\title{
BIOCHEMICAL AND HISTOLOGICAL EVALUATION OF DIFFERENT INTRA-ARTICULAR INJECTIONS AS THERAPY FOR TEMPOROMANDIBULAR JOINT OSTEOARTHRITIS IN RATS
}

\author{
Shereen Wagdy Arafat* and Samah Mohamed Kamel**
}

\begin{abstract}
Introduction: Temporomandibular joint (TMJ) osteoarthritis is usually treated by intraarticular injection of medications. Intra-articular hyaluronic acid (HA) injection reduces friction and stress on the joint cartilage through lubrication, chondro-protection and proteoglycans synthesis. Platelet Rich Plasma (PRP) with its high concentration of growth factors plays a great role in joint repair through enhancing osteo-inductivity, and chondrogenic differentiation. This study aims at comparing intra-articular injection of combination of hyaluronic acid and platelet rich plasma to intra-articular injection of each of them alone.
\end{abstract}

Materials and Methods: 24 young adult male 250-300 grams albino rats were used in the current study divided randomly into 4 groups: Group 1 (control) in which animals were left without treatment. Group 2 which was subdivided into 3 study groups, in Group 2A: the animals received 2 doses of HA, in Group 2B: the animals received 2 doses of PRP, and in Group 2C: the animals received 2 doses of combined PRP and HA. After 28 days, the animals were sacrificed and histological analysis was performed. Synovial fluid Samples were extracted before each intraarticular injection, the concentration of vascular endothelial growth factor (VEGF) and fibroblast growth factor-2 (FGF-2) were detected and statistically analyzed.

Results: Group 2C showed superior findings to the other 2 study groups regarding fibrocartilage and bone regeneration, while Group 2B was superior to group 2A regarding the study parameters.

Conclusion: Intra-articular injection of combination of PRP and HA is superior to intraarticular injection of HA or PRP alone for the treatment of TMJ osteoarthritis in rats.

KEYWORDS: Osteoarthritis, Hyaluronic acid, Platelet Rich Plasma

Associate Professor, Oral Surgery Department, Faculty of Dentistry, MSA University

** Associate Professor, Oral Biology Department, Faculty of Dentistry, MSA University 


\section{INTRODUCTION}

The tempro-mandibular joint (TMJ) is one of the most common joints affected by osteoarthritis (OA), and TMJ-OA is considered the most serious subtype of TMJ disorders (TMD). ${ }^{(1)}$ It was found that Osteoarthritis is present in 10-17\% of patients with TMJ pain. ${ }^{(2)}$ TMJ-OA is a chronic Progressive degenerative disease characterized by degradation and abrasion of the articular cartilage, subchondral bone remodeling, synovitis, and chronic pain. ${ }^{(3,4)}$

The etiology of TMJ-OA is complex, and multifactorial. It was reported that excessive mechanical loading of normal articular cartilage or normal mechanical loading on impaired cartilage usually begins the degenerative process of OA. Moreover, degeneration of cartilaginous extracellular matrix and the catabolic activity causes damage to the cartilage. ${ }^{(5,6)}$ Furthermore, Synovial analysis can provide information about the progression of TMJOA with expression of Pro-inflammatory cytokine levels, including interleukin (IL)-1b, interleukin 6 and tumor necrosis factor (TNF)-a. ${ }^{(7-9)}$

Treatment of TMJ-OA involves noninvasive modalities (which are physical therapy, occlusal splints, non-steroidal anti-inflammatory medications) minimally invasive (arthrocentesis and/or intra-articular injection) and surgical modalities (arthroplasty, condylectomy). ${ }^{(10)}$

The majority of TMJ-OA is usually treated by noninvasive options with intra-articular injections of medications to manage pain, improve the inflammatory state of the joint cavity, slow disease progression, promote condylar cartilage, enhance sub-chondral tissue regeneration, and restore function. Among the substances that have been injected in TMJ-OA are platelet-rich plasma (PRP), and Sodium Hyaluronate. ${ }^{(11)}$

Intra-articular injection of sodium hyaluronate was first described by Rydell and Balazs ${ }^{(12)}$ in 1971 for the treatment of knee osteoarthrosis.
Recently, it has been proposed for the treatment of TMJ-OA. ${ }^{(13,14)}$ It was found that intra-articular hyaluronic acid (HA) injections reduces friction and stress on the joint cartilage through contributing in lubrication, chondro-protection, proteoglycans synthesis and maintain TMJ homeostasis. Moreover, it has great effect in relieving TMJOA symptoms through anti-inflammatory actions by reducing the production and activity of pro-inflammatory mediators. ${ }^{(15)}$

Since 1990, investigators have reported intra-articular injection of platelet-rich plasma (PRP). PRP, an autologous blood product, contains 3 to 8 times the number of platelets found in blood. (16) These concentrated platelets contain multiple growth factors as platelet-derived growth factor, transforming growth factor, epidermal growth factor, basic fibroblast growth factor, and insulin like growth factor. These factors drive fibroblasts in TMJ synovium to secrete HA, and maintain HA levels for joint lubrication and cartilage protection. ${ }^{(17,18)}$

Moreover, PRP contains 3 proteins (fibrin, fibronectin, and vitronectin) which in turn allow osteo-conduction and act as a matrix for migration of bone, cartilage, and epithelium. Furthermore, PRP helps joint repair through enhancing osteoinductivity, and chondrogenic differentiation. ${ }^{(19,20)}$

It was reported that vascular endothelial growth factor (VEGF) and fibroblast growth factor-2 (FGF2) have an important role in chronic inflammatory states as angiogenic factors in the synovial tissues of TMJ. The blood vessels maintain the chronic inflammation by transporting inflammatory cells and supplying nutrients and oxygen to the inflamed tissues. ${ }^{(21,22)}$

Most previous studies have focused on the therapeutic effects of PRP and HA in the treatment of TMJ-OA but few studies have focused on the efficacy and treatment mechanism of a combination of PRP and HA. ${ }^{(23-25)}$ Recently, increasing evidence has supported the use of PRP combined with HA for 
the treatment of knee osteoarthritis. ${ }^{(26,27)}$ Marmotti et al. ${ }^{(28)}$ and Yan et al. ${ }^{(29)}$ found that the addition of HA to PRP could effectively promote the proliferation of chondrocytes and improve cartilage repair. Therefore, the goal of this study was to evaluate the effectiveness of PRP combined with HA for the treatment of TMJ-OA.

\section{MATERIALS AND METHODS}

24 young adult male Albino rats aged about 8 weeks, and weighing 250-300 g were included in the current study. The animals were housed in clean metal cages at the animal house of the Medical Research Center of MSA University, Egypt, with a light-dark cycle (L: D 12:12) in room temperature of 22 to $24 \mathrm{C}$. They were maintained on a regular diet and drinking tap water throughout the duration of the experiment.

\section{Experimental Groups}

The rats were randomly divided into Group 1 (control) and Group 2 which was subdivided into 3 study groups. After induction of OA at day 0 , in Control group $(n=6)$ the animals were left without treatment, in Group 2A (TMJ-HA) $(n=6)$ the animals received 2 doses of $\mathrm{HA}$ at days 7, 21, in Group 2B (TMJ-PRP) $(\mathrm{n}=6)$ The animals received 2 doses of PRP at days 7, 21, and Group 2C (TMJHA-PRP) (n=6) the animals received 2 doses of combined PRP and HA at days 7, 21.

\section{Induction of TMJ-OA}

After shaving the skin over the area of the TMJ for each animal, the joint was felt 5-10 mm posterior to the lateral canthus of the eye, while the mandible and the zygomatic arch of the temporal bone in front of the ear were palpated. The sterile 30 -gauge needle was positioned on the posteroinferior edge of the zygomatic arch (the roof of the joint fossa) and passed through the posterolateral edge of the mandibular condyle toward the antero-medial direction to reach the superior joint cavity. ${ }^{(30)}$

OA was induced in the animals TMJs on day 0 by a unilateral right side single intra-articular injection of $50 \mu \mathrm{l}$ of Complete Freund's adjuvant (CFA) (5881; Sigma-Aldrich, USA), diluted 1:1(oil: saline). ${ }^{(31-33)}$ The animals received $300 \mathrm{mg} /$ $\mathrm{kg}$ Acetaminophen (Paracetamol) added in drinking water after OA induction to decrease pain. The contralateral left side TMJ in each study animal was left normal not to compromise animal feeding.

\section{PRP Preparation}

$5 \mathrm{ml}$ Peripheral blood was collected from the Marginal ear vein into glass tubes containing anticoagulant acid citrate dextrose (Corning, Lowell, MA). At room temperature, the blood was centrifuged (TGL-16Gr, Anting Scientific Instrument Factory, Shanghai, China) at $160 \mathrm{~g}$ for 10 minutes. The blood was separated into 3 components: erythrocytes at the bottom, a buffy coat in the middle, and platelet containing plasma at the top. ${ }^{(34)}$ (Medifuge MF200, Italy Silfdradent). The platelet-containing plasma was then aspirated and transferred to a new tube without anticoagulant and centrifuged at $250 \mathrm{~g}$ for 15 minutes. The platelet poor supernatant plasma was aspirated and discarded. PRP was carefully drawn into a separate syringe for intra-articular injection. ${ }^{(35)}$

\section{Intra-articular injections}

One week following the induction of OA, In group 2A (TMJ-HA) $(n=6)$, the animals received 2 intra-articular slow injections of $50 \mu$ high molecular weight $\mathrm{HA}$ at days 7,21 . In group $2 \mathrm{~B}$ (TMJ-PRP) $(\mathrm{n}=6)$ : the animals received 2 slow intra-articular injections of $50 \mu 1$ freshly prepared PRP at days 7, 21. While in group C (TMJ-HAPRP) $(n=6)$ : the animals received a combination of $50 \mu 1 \mathrm{HA}$ and PRP slow intra-articular injections at days 7,21 . 


\section{Biochemical Analysis}

Samples of the TMJ synovial fluid were extracted from each animal at days 7,21 before the intra-articular injection and at day 28 before scarification and stored at $-80 \mathrm{C}$. The concentration of vascular endothelial growth factor (VEGF) and fibroblast growth factor-2 (FGF-2) were detected using an enzyme-linked immune-sorbent assay (ELISA) kit according to the manufacturer's instructions. The minimum detectable level of VEGF is $<10 \mathrm{pg} / \mathrm{mL}$. The minimum detectable level of FGF-2 is $<5 \mathrm{pg} / \mathrm{mL}$.

All Control and study animals of the current study were sacrificed at day 28 after induction of OA through inhalation of an overdose of (Diethyl ether) under veterinary control.

\section{Histological \& histo-chemical Preparations}

The TMJs were dissected. The bone was bur milled under irrigation, and the condylar process of the mandible were excised. Samples were fixed with $10 \%$ neutral formalin, decalcified in $10 \%$ ethylene-diamine-tetra-acetic acid (EDTA) for 10 weeks, then embedded in paraffin, and stained with Hematoxylin and Eosin (H\&E) as a Routine stain for cellular details. In addition, Toluidine blue Stain was utilized for optimal demonstration of mineralized bone and chondrocytes.

\section{Statistical Analysis}

Data showed parametric distribution when checked for normality using Shapiro-Wilk test. Three-way ANOVA used to show the effect of concentration, groups, and follow-up. Multiple comparisons were performed with Tukey HSD. Significant level was set at $\mathrm{p}=0.05$.

\section{RESULTS}

All intra-articular injection procedures went well without adverse event that required study cessation. Synovial fluid samples were successfully withdrawn from all rats TMJ in the control and study groups at the study intervals.

\section{Histological and histo-chemical findings}

At the end of the current study, By examining the head of the condyles of the control and study groups, Group 1 (control group) showed loss of the fibro-cartilaginous layer, irregular bone surface and widening of the bone marrow space (Fig. 1A), Group 2A (TMJ-HA) showed slight incomplete regeneration of the fibro-cartilaginous layer, the underlying bone structure was regenerated and the bone marrow spaces were decreased (Fig. 1B), Regeneration was better in group 2B (TMJ-PRP), the fibro-cartilaginous layer was covering all the condylar head while the thickness was deficient, the underlying bone structure showed normal architecture. (Fig. 1C) The regeneration of the fibro-cartilaginous layer was completed and the underlying bone was normal in group 2C (TMJHA-PRP). (Fig. 1D)

At higher magnification By $H \& E$ stain, the condyle of Group 1 (control group) showed loss of the fibrous layer covering it, the cartilaginous layer was destructed, the chondrocytes were abnormal as they were vacuolated with eccentric nuclei, and the underlying bone showed widened bone marrow spaces. (Fig 2A) By toluidine blue stain, the vacuolated chondrocytes were noticed, the bone trabeculae were very thin and pale blue indicating being less mineralized. (Fig 2B)

In Group 2A(TMJ-HA) the covering fibrous layer of the fibro-cartilaginous layer was absent is some areas, in other parts the fibrous layer was regenerated as fibroblasts and collagen fibers were noticed, the cartilaginous layer showed chondrocytes, and some area showed hyalinization (Fig.3)

By using toluidine blue, Group 2A (TMJ-HA) showed the chondrocytes in their lacunae in the cartilaginous layer, bone marrow spaces were normal. The bone trabeculae were regenerated but they exhibited pale blue stain denoting hypomineralization. (Fig. 4) 


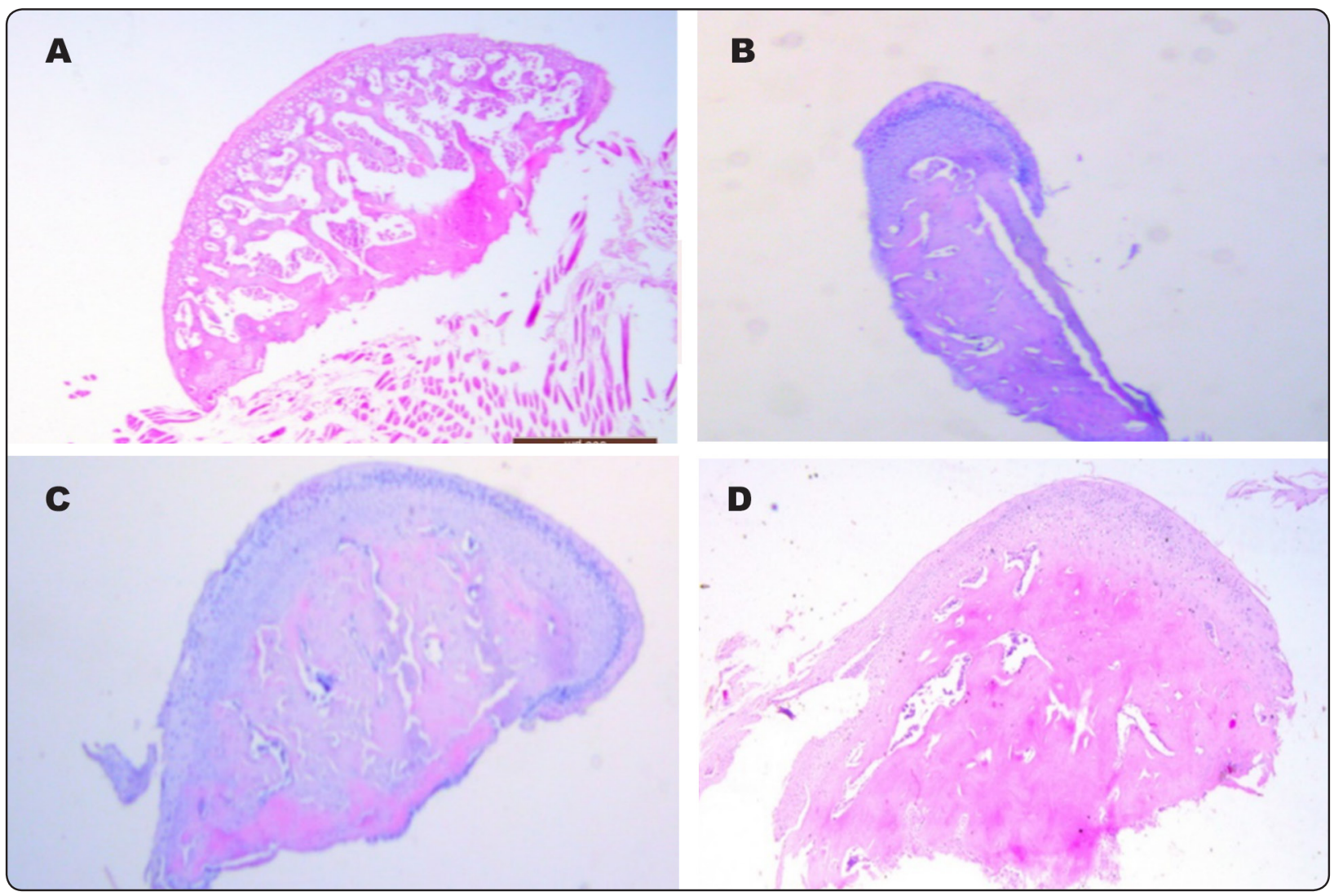

Fig. (1) Photomicrograph showing that The fibro-cartilaginous layer was absent in Group 1 with large marrow spaces (A), the fibrocartilaginous layer started to regenerate in group 2A (B), in group 2B the layer was completed but thin (C), and complete regeneration was noticed in group $2 \mathrm{C}(\mathrm{D})(\mathrm{H} \& \mathrm{E} \mathrm{X} 40)$

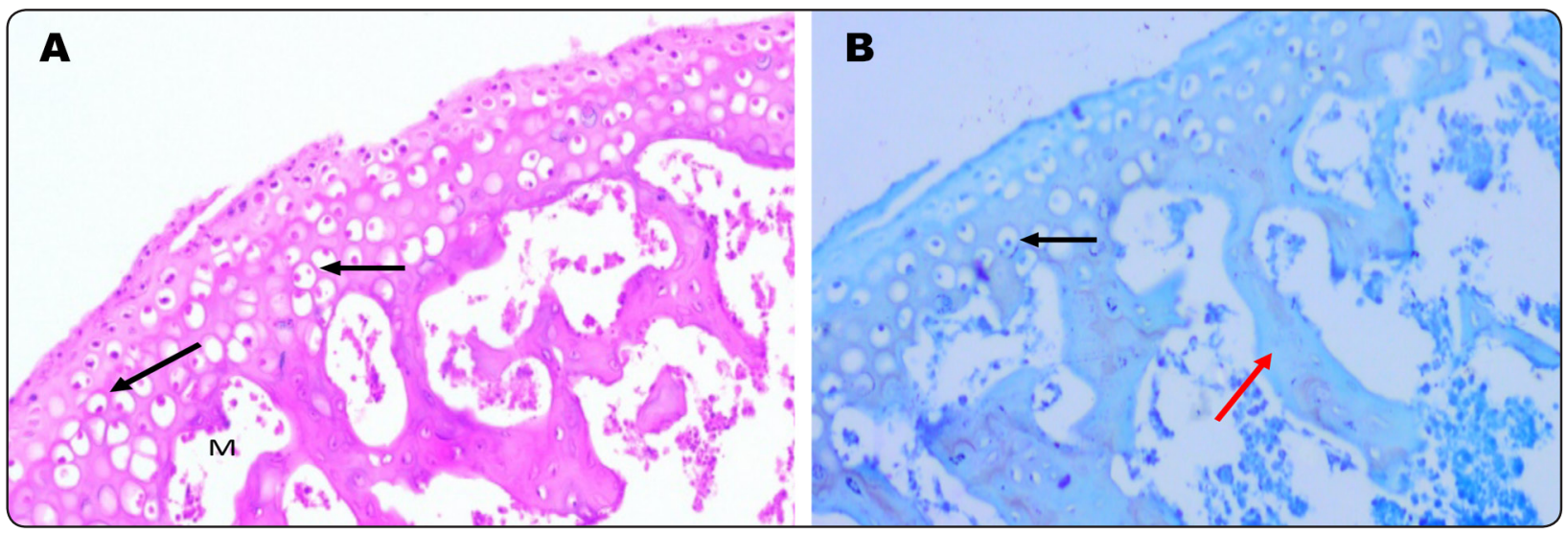

Fig (2) : Photomicrograph (Group 1: control) showing that chondrocytes are vacuolated ( black arrows), the marrow spaces are widened ( M), the bone trabeculae are pale blue and thin ( Red arrow) (2A H\&E X200, 2B Toluidine X 200) 


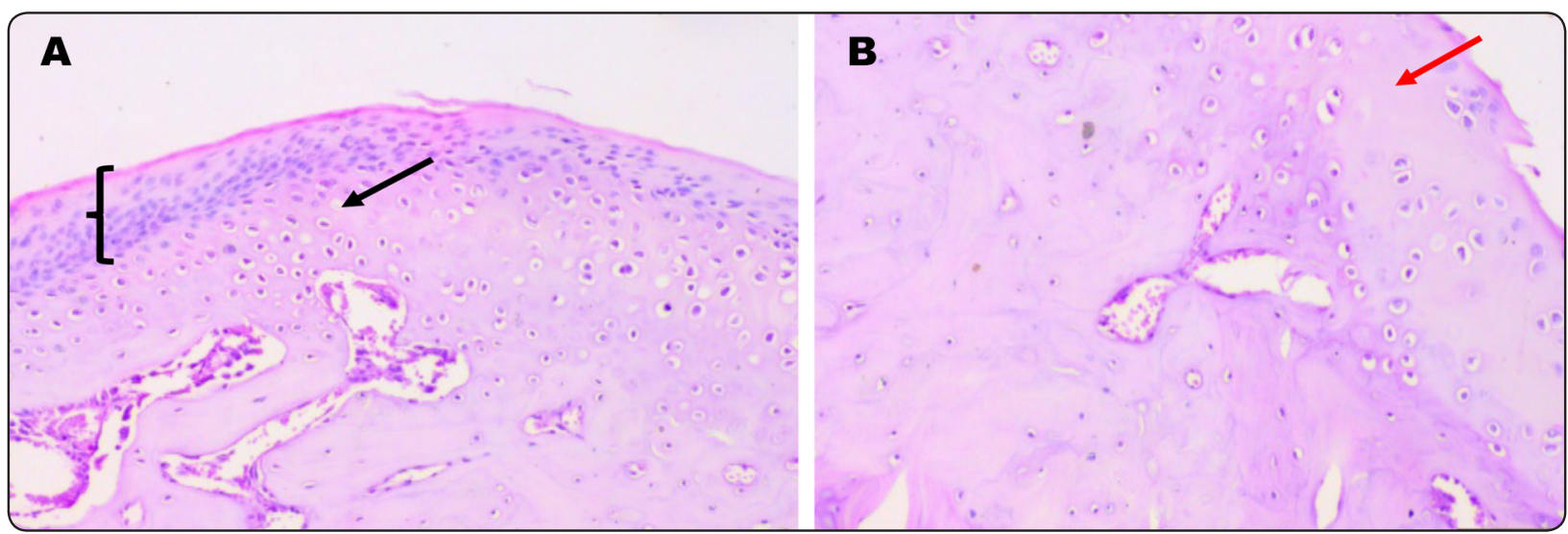

Fig. (3) : Photomicrograph (group 2A: TMJ-HA) showing regenerated thin fibrous layer containing fibroblasts(black bracket), normal appearance of the chondrocytes ( black arrow), in B absence of fibrous layer and areas of hyalinization (red arrow) were noticed (H\&E X200)

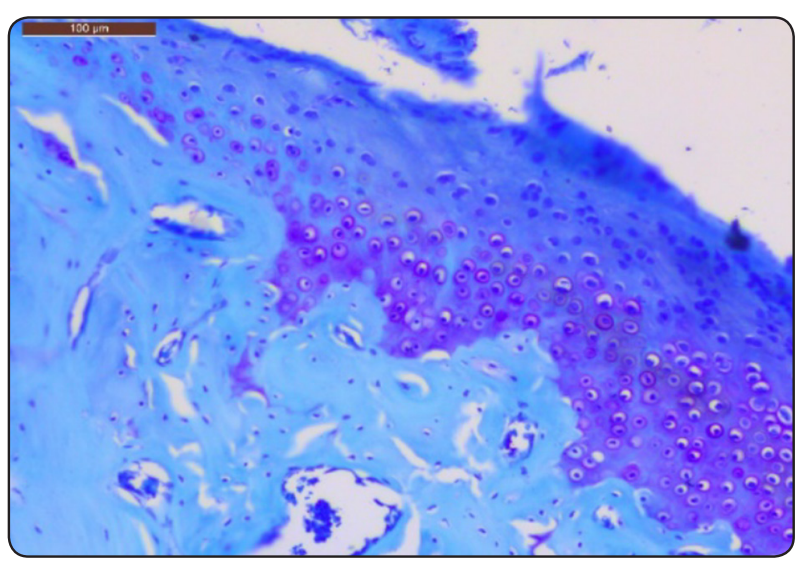

Fig. (4) : Photomicrograph (group2A: TMJ-HA) showing the regeneration of the cartilaginous layer, the underlying bone structure is regenerated but still less mineralized than normal ( Toluidine X 200)
In Group 2B (TMJ-PRP) the fibro-cartilaginous layer was fully formed with thick well-formed cartilaginous layer. Moreover, the fibrous layer was found in all the parts of the condyle but was very thin in some areas while its surface was not smooth. The underlying bone showed normal architecture. (Fig. 5)

By using Toluidine blue, Group 2B (TMJ-PRP) showed normal fibro-cartilaginous layer with thin fibrous layer, normal bone architecture with better mineralization than other groups. (Fig. 6)

In Group 2 C (TMJ-HA-PRP), the fibro-cartilaginous layer was fully formed with smooth surface, fibrous part was fully formed and thicker than other groups with normal bone structure. (Fig. 7)

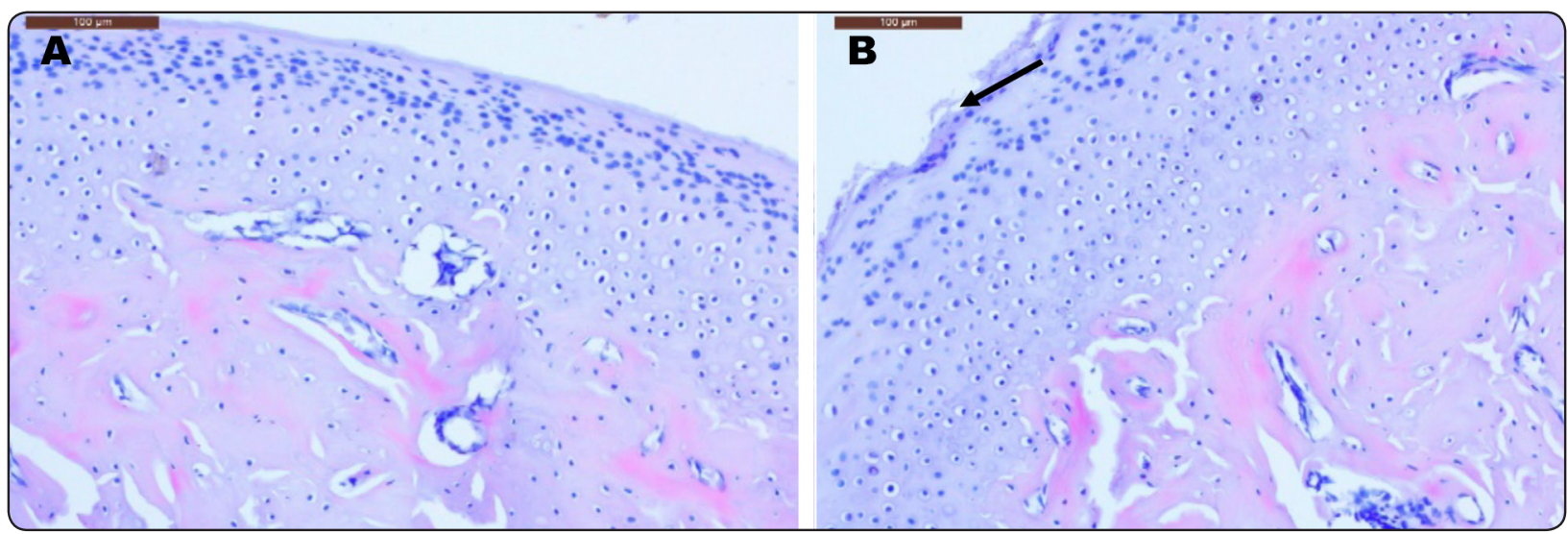

Fig. (5): Photomicrograph (group 2B:TMJ-PRP) showing regenerated fibro-cartilaginous layer normal chondrocytes , thin fibrous layer irregular in some areas (black arrow), normal bone structure (H\&E X 200) 


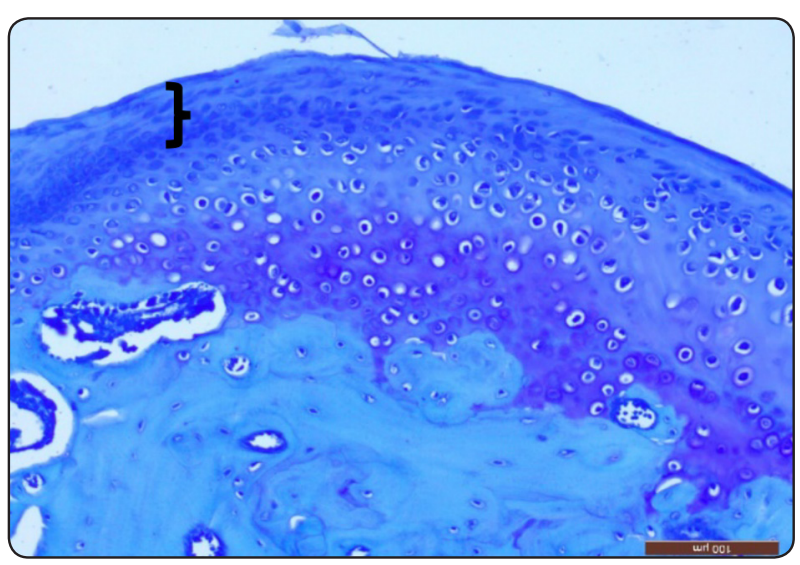

Fig 6: Photomicrograph (group 2B:TMJ-PRP) showing fibro-cartilaginous layer with thin fibrous part (black bracket), normal underlying bone structure (Toluidine X 200)

\section{Biochemical analysis findings:}

There was no difference between the tested groups at day 7 (before intra-articular injections). By the end of the current study at day 28 , group $2 \mathrm{C}$ (TMJ-HA-PRP) showed significant lower values of VEGF/FGF-2 compared to the other 2 study groups.

For both FGF-2 and VEGF, group 2A (TMJ-HA) showed a significant reduction after 28 days while group 2B (TMJ-PRP) showed a significant reduction after 21 days followed an insignificant reduction after 28 days. Group 2C (TMJ-HA-PRP) showed a significant reduction after 21 days followed by a further significant reduction after 28 days.

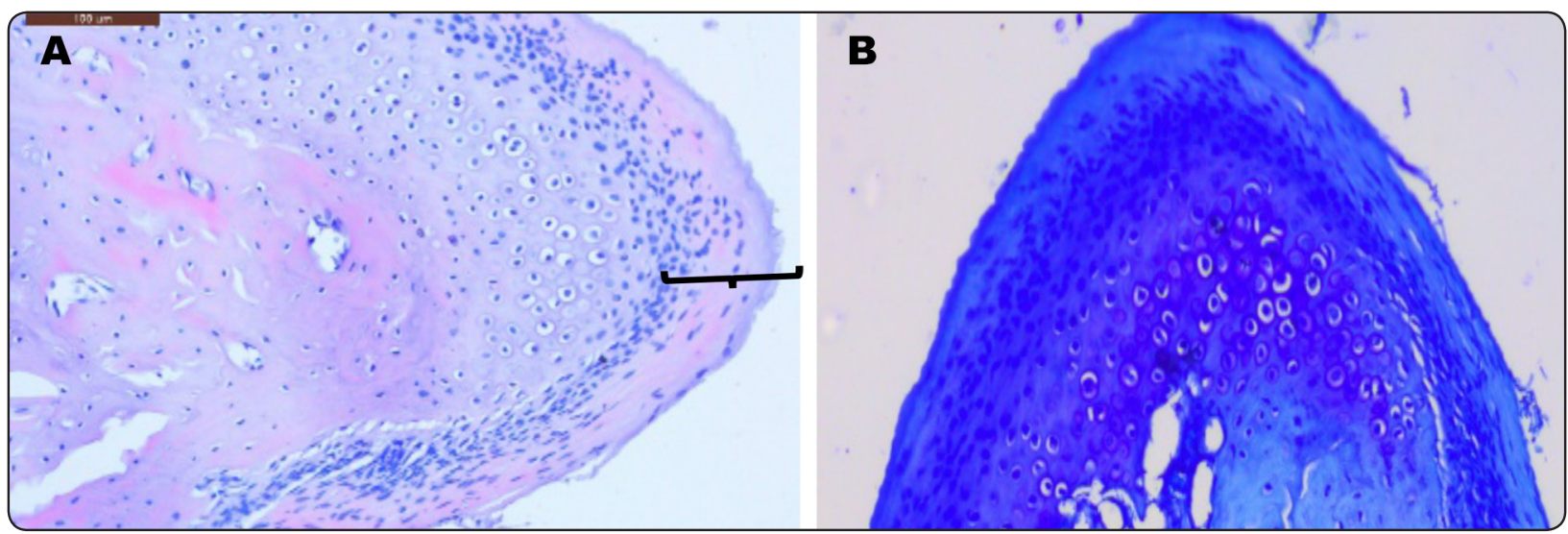

Fig 7: Photomicrograph (group 2C:TMJ-HA-PRP) showing fibro-cartilaginous layer with thick fibrous part (black bracket), normal underlying bone structure (7A H\&E X 200, 7B Toluidine X200)

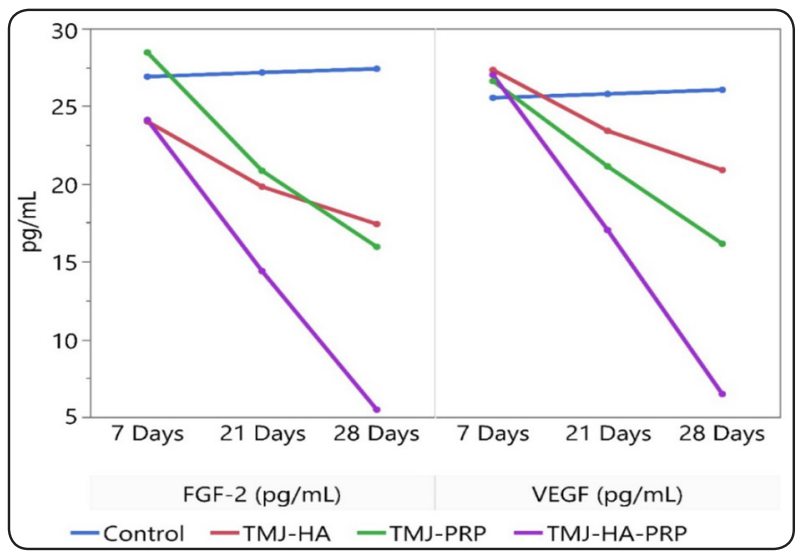

Fig. 8: Line chart showing the comparison of VEGF/FGF-2 concentrations for the control and study groups among the study intervals.

\section{DISCUSSION}

In the current study, OA was induced in the right TMJs of 24 albino rats so as not to compromise the animal feeding. Rats were selected based on the similarity of the condylar translatory movements to those of human condyle, rapid skeletal maturation in comparison with larger animals, and easy handling during OA induction. ${ }^{(36,37)}$ The current study period was selected to be 28 days as the TMJ induced arthritis using CFS has proved to remain for 6 weeks with elevated inflammatory mediators concentrations as reported by Spears et al. ${ }^{(38)}$ 
High molecular weight HA was used in the current study as it usually requires smaller number of intra-articular injection and provides prolonged positive effects than low molecular weight HA as recommended by previous studies. ${ }^{(39,40)}$

This study demonstrated that intra-articular injection of combination of PRP and HA was superior to intra-articular injection of HA or PRP alone regarding fibro-cartilaginous regeneration and decreased concentration of angiogenic factors (VEGF/ FGF-2). Moreover, intra-articular injection of PRP was in turn superior to intra-articular injection of HA regarding the study parameters. However, all the study groups showed improvement of OA after two injections regarding the study parameters.

The improvement reported in the current study regarding intra-articular HA injection in group 2A is in agreement with Radin et al ${ }^{(41)}$ who reported that HA has an essential role in synovial tissue lubrication. Also, Gotoh et al ${ }^{(42)}$, and Cascone et al ${ }^{(43)}$ reported that HA prevents intra-articular adhesions through lubrication and feeding the joint components. Moreover, Kim et al ${ }^{(44)}$ reported that HA injection stimulates synovial cells to produce natural HA. Collaborating our findings, Lemos G. ${ }^{(45)}$ concluded in a histologic study on TMJ induced $\mathrm{OA}$ in rats that HA reduced the histologic changes and led to superior arrangement of collagenous fibers, and suggested that HA may exert a protective effect on the TMJ.

The current study reported proper histological and biochemical findings after intra-articular injection of PPR in group 2B. This finding is in agreement with Haigler et al ${ }^{(46)}$ who reported that PRP injection to treat TMJ OA could stimulate the regeneration of damaged tissues. Moreover, it was reported by several studies that PRP proved to improve cartilage repair in degenerative knee pathologic disorder. ${ }^{(47,48)}$ Furthermore, Takafuji et al ${ }^{(49)}$ showed the therapeutic great effect of PRP growth factors for TMJ articular cartilage defects treatment. This regenerative effect of PRP could be attributed to the great amount of growth factors released which in turn stimulate the proliferation and differentiation of fibroblasts, osteoblasts, chondrocytes, and mesenchymal stem cells. ${ }^{(50)}$ Also, Wu et al ${ }^{(51)}$ proved that PRP plays a critical role in regulating cell proliferation, chondrogenesis and inflammatory gene expression of articular chondrocytes.

However, Kutuk et al ${ }^{(52)}$ reported in their study that the healing of fibrocartilage and hyaline cartilage was not significantly better in the PRP group than in the control group. This could be explained by the severe degenerative changes induced in their study by creating surgical defects.

The current study showed that Intra-articular injection of PRP was superior to HA in the study parameters. This finding corresponds to that of Spakova et al ${ }^{(53)}$ who reported significantly better results of PRP than HA for treatment of 120 patients with knee osteoarthritis. Moreover, Gormelli et al ${ }^{(54)}$ reported in their study that PRP injections showed better clinical results than $\mathrm{HA}$ as intra-articular injection for treatment of knee osteoarthritis.

This study demonstrated that PRP combined with HA had excellent results regarding fibro-cartilaginous healing which was superior to HA or PRP alone. This finding agrees with that of $\mathrm{Xu}$ et al ${ }^{(55)}$ and Lana et al ${ }^{(56)}$ who reported that this combination was more effective than PRP or HA alone at inhibiting synovial inflammation and effectively improved pain and function in knee osteoarthritis.

The biochemical assessment showed significant more decrease in the concentration of VEGF/FGF2 in TMJ-HA-PRP group than the other groups by the end of the study which in turn supports the histologic and histochemical findings of the current study. This finding was in accordance with those of Kumagai ${ }^{(57)}$ who reported that the level of VEGF in TMJ synovial fluid seems to reflect the clinical status in patients with synovitis. 
The current study showed that for both FGF2 and VEGF, group 2A (TMJ-HA) showed a significant reduction after 28 days while group $2 \mathrm{~B}$ (TMJ-PRP) showed a significant reduction after 21 days. This finding support as well the histologic finding that TMJ-PRP group was more superior to TMJ-HA group. On the other hand, Wang et el ${ }^{(58)}$ reported that VEGF was significantly higher before treatment with HA than after treatment, but there was no significant difference in the concentration of FGF-2 between before and after treatment. This could be explained by only one time point of observation that was applied after the HA injection.

In conclusion, our study has shown that intraarticular injection of combined HA-PRP has potential beneficial effects on fibrocartilage and bone regeneration in experimentally induced osteoarthritis in rats. Moreover, this combination has significant effect on reducing the concentrations of VEGF/FGF-2. Additional research evaluating the effects of HA-PRP on human TMJ with osteoarthritis with long term results and functional outcomes is needed for clinical application.

\section{REFERENCES}

1. Liu, Q., Yang, H., Zhang, M., Zhang, J., Lu, L., Yu, S., $\mathrm{Wu}, \mathrm{Y}$. , and Wang, M.: Initiation and progression of dental-stimulated temporomandibular joints osteoarthritis. Osteoarthritis and Cartilage, 12:16-21, 2020.

2. Siddharth, K.: TMJ osteoarthritis and early diagnosis. Journal of Oral Biology and Craniofacial Research, 3:109110, 2013.

3. Mercuri, L.: Osteoarthritis, osteoarthrosis, and idiopathic condylar resorption. Oral Maxillofac. Surg. Clin. North Am., 20: 169-183, 2008.

4. Tanaka, E., Detamore, M., and Mercuri, L.: Degenerative disorders of the temporomandibular joint: etiology, diagnosis, and treatment. J. Dent. Res., 87: 296-307, 2008.

5. Aigner, T., Soeder, S., and Haag, J.: IL-1beta and BMPsinteractive players of cartilage matrix degradation and regeneration. Eur. Cell Mater., 12:49-52, 2006.

6. Loeser, R., Olex,A., and McNulty, M.: Disease progression and phasic changes in gene expression in a mouse model of osteoarthritis. J.PLOS One, 8(1) e54633, 2013.
7. Yang, M., Wang, D., and Wu, H.: Correlation of magnetic resonance imaging grades with cytokine levels of synovial fluid of patients with temporomandibular joint disorders: a cross-sectional study. Clin. Oral Investig., 23:3871-8, 2019.

8. Kristensen, K., Alstergren, P., and Stoustrup, P.: Cytokines in healthy temporomandibular joint synovial fluid. J. Oral Rehabil., 41:250-6,2014.

9. Ernberg, M.: The role of molecular pain biomarkers in temporomandibular joint internal derangement. J. Oral Rehabil., 44:481-91,2017.

10. De Souza, R., Lovato da Silva, C., Nasser, M., Fedorowicz, Z., and Al-Muharraqi, M.: Interventions for the management of temporomandibular joint osteoarthritis. Cochrane Database Syst. Rev., CD007261, 2012.

11. Wang, X., Zhang, J., and Gan, Y.: Current understanding of pathogenesis and treatment of TMJ osteoarthritis. J. Dent. Res., 94:666-673, 2015.

12. Rydell, N., and Balazs, E.: Effect of intra-articular injection of Hyaluronic acid on the clinical symptoms of osteoarthritis and on granulation tissue formation. Clin. Orthop., 80: 25-32, 1971.

13. Guarda-Nardini, L., Masiero, S., and Marioni, G.: Conservative treatment of temporomandibular joint osteoarthrosis: intra-articular injection of Hyaluronic acid. J. Oral Rehabil., 32: 729-734, 2005.

14. Guarda-Nardini, L., Tito, R., Staffieri, A., and Beltrame, A.: Treatment of patients with arthrosis of the temporomandibular joint by infiltration of Hyaluronic acid: a preliminary study. Eur. Arch Otorhinolaryngol., 259: 279-284, 2002.

15. Altman, R., Manjoo, A., Fierlinger, A., and Niazi, F.: The mechanism of action for hyaluronic acid treatment in the osteoarthritic knee: A systematic review. BMC Musculoskeletal Disorders, 16(1), 321, 2015.

16. Zhu, Y., Yuan, M., and Meng, H.: Basic science and clinical application of platelet-rich plasma for cartilage defects and osteoarthritis: a review. Osteoarthritis Cartilage, 21(11):1627-1637, 2013.

17. Anitua, E., Sanchez, M., and Nurden, A.: Platelet-released growth factors enhance the secretion of hyaluronic acid and induce hepatocyte growth factor production by synovial fibroblasts from arthritic patients. Rheumatol. 46:1769, 2007.

18. Peerbooms, J., Sluimer, J., and Bruijn, D.: Positive effect of an autologous platelet concentrate in lateral epicondylitis in a double-blind randomized controlled trial: Plateletrich plasma versus corticosteroid injection with a 1-year follow-up. Am. J. Sports Med., 38:255, 2010. 
19. Cömert, K., Gungormus, M., and Sumbullu, M.: Is arthrocentesis plus platelet-rich plasma superior to arthrocentesis alone in the treatment of temporomandibular joint osteoarthritis: a randomized clinical trial. J. Oral Maxillofac. Surg., 73(8):1473-1483, 2015.

20. Marx, R.: Platelet-rich plasma: evidence to support its use. J. Oral Maxillofac. Surg., 62(4):489-496, 2004.

21. Jackson, J., Minton, J., Ho, M., Wei, N., and Winkler, $\mathrm{J}$.: Expression of vascular endothelial growth factor in synovial fibroblast is induced by hypoxia and interleukin 1 beta. J. Rheumatol., 24:1253-1259, 1997.

22. Koch, A., Harlow, L., Haines, G., Amento, E., Unemori, E., and Wong, W.: Vascular endothelial growth factor. A cytokine modulating endothelial function in rheumatoid arthritis. Am. J. Immunol., 152:4149-4156, 1994.

23. Fu-Long, L., Chuan-Bin, W., Hai-Jiang, S., and Qing, Z.: Comparison of Autologous Platelet-Rich Plasma and Chitosan in the Treatment of Temporomandibular Joint Osteoarthritis: A Retrospective Cohort Study. J. Oral Maxillofac. Surg., 1-9, 2020.

24. Iturriaga, V., Bornhardt, T., Manterola, C., and Brebi, P.: Effect of hyaluronic acid on the regulation of inflammatory mediators in osteoarthritis of the temporomandibular joint: a systematic review. Int. J. Oral Maxillofac. Surg. 46: 590595,2017

25. Tolbaa, M., Omara, S., Naguia, D., and Nawwarb, M.: Effect of high molecular weight hyaluronic acid in treatment of osteoarthritic temporomandibular joints of rats. Archives of Oral Biology, 110:104618, 2020.

26. Yu, W., Xu, P., and Huang, G.: Clinical therapy of hyaluronic acid combined with platelet-rich plasma for the treatment of knee osteoarthritis. Exp. Ther. Med., 16:21192125, 2018.

27. Chen, S., Kuan, T., and Kao, M.: Clinical effectiveness in severe knee osteoarthritis after intra-articular plateletrich plasma therapy in association with hyaluronic acid injection: three case reports. Clin. Interv. Aging., 11: 12131219, 2016.

28. Marmotti, A., Bruzzone, M., and Bonasia, D.: Onestep osteochondral repair with cartilage fragments in a composite scaffold. Knee Surg. Sports. Traumatol. Arthrosc., 20:2590-601, 2012.

29. Yan, W., Xu, X., and Xu, Q.: Platelet-rich plasma combined with injectable hyaluronic acid hydrogel for porcine cartilage regeneration: A 6-month follow-up. Regen. Biomater., 7:77-90, 2020.
30. Yuce, P., Hosgor,H.,Rencber, S., and Yazir, Y.: Effects of Intra-Articular Resveratrol Injections on Cartilage Destruction and Synovial Inflammation in Experimental Temporomandibular Joint Osteoarthritis. J. Oral Maxillofac. Surg., 1:1-12, 2020.

31. Harper, R., Kerins, C., McIntosh, J., Spears, R., and Bellinger, L.: Modulation of the inflammatory response in the rat TMJ with increasing doses of complete Freund's adjuvant. Osteoarthritis and Cartilage, 9(7), 619-624, 2001.

32. George, M., Owen, C., Reinhardt, A., Giannini, P., Marx, D., and Reinhardt, R.: Effect of simvastatin injections on temporomandibular joint inflammation in growing rats. J. Oral Maxillofac. Surg., 71(5), 846-853, 2013.

33. Lemos, G., Rissi, R., de Souza, P.,ires, de Oliveira, L., de Aro, A., Pimentel, E., and Palomari, E.: Low-level laser therapy stimulates tissue repair and reduces the extracellular matrix degradation in rats with induced arthritis in the temporomandibular joint. Lasers in Medical Science, 31(6), 1051-1059, 2016.

34. Xu,Z., He, Z., Shu, L., Li, X., Ma,M., and Ye, C.: IntraArticular Platelet-Rich Plasma Combined With Hyaluronic Acid Injection for Knee Osteoarthritis Is Superior to Platelet-Rich Plasma or Hyaluronic Acid Alone in Inhibiting Inflammation and Improving Pain and Function. J. Arthros. \& Rel. Surg., 1-13, 2020.

35. Yin, W., Xu, H., and Sheng, J.: Optimization of pure platelet rich plasma preparation: A comparative study of pure platelet-rich plasma obtained using different centrifugal conditions in a single-donor model. Exp. Ther. Med. 14: 2060-2070, 2017.

36. El-Hakim, I., Abdel-Hamid, I., and Bader, A.: Tempromandibular joint (TMJ) response to intra-articular dexamethasone injection following mechanical arthropathy: a histological study in rats. Int. J. Oral Maxillofac. Surg., 34: 305-310, 2005.

37. Kalladka, M., Quek, S., Heir, G., Eliav, E., Mupparapu, M., and Viswanath, A.: Temporomandibular joint osteoarthritis: diagnosis and long-term conservative managementda topic review. J. Indian Prosthodont. Soc., 14(1): 6-15, 2014.

38. Spears, R., Dees, L., Sapozhnikov, M., Bellinger, L., and Hutchins, B.: Temporal changes in inflammatory mediator concentrations in an adjuvant model of temporomandibular joint inflammation. J. Orofac. Pain, 19(1), 2005.

39. Hsieh, Y., Yang, S., Lue, K., Chu, S., and Lu, K.: Effects of different molecular weight hyaluronan products on the expression of urokinase plasminogen activator and inhibitor and gelatinases during the early stage of osteoarthritis. J. Orthop. Res., 26(4), 475-484, 2008. 
40. Duygu, G., Güler, N., Cam, B., and Kürkçü, M.: The effects of high molecular weight hyaluronic acid (Hylan G-F 20) on experimentally induced temporomandibular joint osteoartrosis: part II. Int. J. Oral Maxillofac. Surg., 40(12), 1406-1413, 2011.

41. Radin, E., Paul, I., Swann, D., and Shottstaedt, E.: Lubrication of synovial membrane. Ann. Rheum. Dis., 30: 322-325, 1971.

42. Gotoh, S., Miyazakik, K., Onaya, J., Sakamoto, T., Tokuyasu, K., and Namiki, O.: Experimental knee pain model in rats and analgesic effect of sodium hyaluronate (SPH). Folia Nippon Yakurigaku Zasshi, 92(1): 17-27, 1988.

43. Cascone, P., Fonzi Dagger, L., and Aboh, I.: Hyaluronic acid's biomechanical stabilization function in the temporomandibular joint. J. Craniofac. Surg., 13: 751-754, 2002.

44. Kim, C., Lee, B., Seo, K., Park, J., Lee, J., Choi, E., Hong, J., Lee, Y., and Park, J.: Therapeutic effect of Hyaluronic acid on experimental osteoarthrosis of Ovine temporomandibular joint. J. Vet. Med. Sci., 63: 1083-1089, 2001.

45. Lemos, G., Rissi, R., Pimentel, E., and Palomari, E.: Effects of high molecular weight hyaluronic acid on induced arthritis of the temporomandibular joint in rats. Acta. Histo-chemica., 117(6), 566-575, 2015.

46. Haigler, M., Abdulrehman,E., Siddappa,S., Kishore,R., Padilla,M., and Enciso,R. : Use of platelet-rich plasma, platelet-rich growth factor with arthrocentesis or arthroscopy to treat temporomandibular joint osteoarthritis Systematic review with meta-analyses. J.A.D.A., 149(11):940-952, 2018.

47. Sampson, S., Reed, M., and Silvers, H.: Injection of platelet-rich plasma in patients with primary and secondary knee osteoarthritis: A pilot study. Am. J. Phys. Med. Rehabil., 12:961, 2010.

48. Kon, E., Buda, R., and Filardo, G.: Platelet-rich plasma: intraarticular knee injections produced favorable results on degenerative cartilage lesions. Knee Surg. Sports Traumatol. Arthrosc., 4: 472, 2010.

49. Takafuji, H., Suzuki, T., and Okubo, Y.: Regeneration of articular cartilage defects in the temporomandibular joint of rabbits by fibroblast growth factor-2: A pilot study. Int. J. Oral Maxillofac. Surg. 10: 934, 2007.
50. Loeser, R., Pacione, C., and Chubinskaya, S.: The combination of insulin-like growth factor 1 and osteogenic protein 1 promotes increased survival of and matrix synthesis by normal and osteoarthritic human articular chondrocytes. Arthritis Rheum., 48: 2188, 2003.

51. Wu, C., Chen, W., and Zao, B.: Regenerative potentials of platelet-rich plasma enhanced by collagen in retrieving proinflammatory cytokine-inhibited chondrogenesis. Biomaterials, 25:5847, 2011.

52. Nukhet, K., Burcu, B., Emrah, S., Zeynep, B., Canay, Y., Esra, B., and Alper, A.: Effect of Platelet-Rich Plasma on Fibrocartilage, Cartilage, and Bone Repair in Temporomandibular Joint. J. Oral Maxillofac. Surg. 72:277-284, 2014.

53. Spakova, T., Rosocha, J., and Lacko, M.: Treatment of knee joint osteoarthritis with autologous platelet-rich plasma in comparison with hyaluronic acid. Am. J. Phys. Med. Rehabil., 91:411, 2012.

54. Görmeli, G., Görmeli, C., and Ataoglu, B.: Multiple PRP injections are more effective than single injections and hyaluronic acid in knees with early osteoarthritis: A randomized, double-blind, placebo-controlled trial. Knee Surg. Sports Traumatol. Arthrosc., 25:958-965,2017.

55. Zhe,X.,Zhixu,H.,Liping, S., Xuanze,L., Minxian,M., and Chuan, Y.: Intra-Articular Platelet-Rich Plasma Combined With Hyaluronic Acid Injection for Knee Osteoarthritis Is Superior to Platelet-Rich Plasma or Hyaluronic Acid Alone in Inhibiting Inflammation and Improving Pain and Function. J. Arthros. Rel. Surg., 1-13, 2020.

56. Lana, J., Weglein, A., and Sampson, S.: Randomized controlled trial comparing hyaluronic acid, platelet-rich plasma and the combination of both in the treatment of mild and moderate osteoarthritis of the knee. J. Stem Cells Regen. Med., 12:69-78, 2016.

57. Kumagai, K., Hamada, Y., and Holmlund, A.: The levels of vascular endothelial growth factor in the synovial fluid correlated with the severity of arthroscopically observed synovitis and clinical outcome after temporomandibular joint irrigation in patients with chronic closed lock. Oral Surg. Oral Med. Oral Pathol. Oral Radiol. Endod., 109:185-90,2010.

58. Wang, X., Fang, W., Li, Y., Long,X., and Cai, H.: Synovial fluid levels of VEGF and FGF-2 before and after intraarticular injection of hyaluronic acid in patients with temporomandibular disorders: a short-term study. Br. J. Oral Maxillofac. Surg., 59: 64-69, 2021. 